\title{
Daseinsvorsorge, gleichwertige Lebensverhältnisse und ländliche Räume im 21. Jahrhundert
}

\author{
Peter Weingarten · Annett Steinführer
}

Online publiziert: 7. Dezember 2020

(C) Der/die Autor(en) 2020

Wann immer von der Gleichwertigkeit der Lebensverhältnisse gesprochen oder geschrieben wird: Ein Bezug zum Thema der Daseinsvorsorge wird nahezu in jedem Falle unmittelbar hergestellt (vgl. z. B. ARL 2016; Steinmeier 2019; Kersten et al. 2020). Meist geht es heute dann ausschließlich um ländliche Räume (früher auch um das sogenannte Zonenrandgebiet), ihre tatsächlichen oder vermuteten Defizite sowie die Sicherung oder den Umbau technischer, sozialer, medizinischer und anderer Einrichtungen der wohnortnahen Versorgung. Zugleich handelte es sich stets um einen Selbstverortungsdiskurs einer Gesellschaft im Wandel, ihres Sozialstaats- und Politikverständnisses. Ein jüngeres Beispiel dafür waren die teils heftigen - ablehnenden wie zustimmenden - Reaktionen auf die Aussage der Bundesforschungsministerin Anja Karliczek im November 2018: ,5G ist nicht an jeder Milchkanne notwendig“ (Reuters 2018). Ist es heute die Digitalisierung, an der Ausstattungsstandards und damit auch die Modernität unterschiedlicher Raumtypen verhandelt werden, waren es noch vor wenigen Jahrzehnten Kanalisation, Straßenbau oder weiterbildende Schulen.

\section{Ländliche Räume im Fokus medialer und politischer Aufmerksamkeit}

Ländliche Räume sind in den letzten Jahren verstärkt in den Fokus medialer und politischer Aufmerksamkeit gerückt (Strotdrees 2018). Auslöser hierfür waren insbesondere die Abstimmungsergebnisse zum Brexit-Referendum und zur US-Präsi-

Prof. Dr. P. Weingarten $(\varangle) \cdot$ Dr. A. Steinführer

Thünen-Institut für Ländliche Räume, Bundesallee 64, 38116 Braunschweig, Deutschland

E-Mail: peter.weingarten@thuenen.de

Dr. A. Steinführer

E-Mail: annett.steinfuehrer@thuenen.de 
dentenwahl 2016 sowie die Wahlergebnisse der AfD bei der Bundestagswahl 2017 (z. B. Ehrenstein 2016; Volkert und Datli 2017). In den Medien wurde seitdem über „abgehängte“ Regionen und über Gefühle des „Abgehängtseins“ berichtet, und dies oft in Zusammenhang mit ländlichen Räumen oder „,dem ländlichen Raum“. Deppisch (2019) wertete in einer Untersuchung 233 mit den Suchbegriffspaaren „AfD, abgehängt“ und ,ländlich, abgehängt“ ermittelte Beiträge aus Print- und Onlinemedien aus dem 2. Halbjahr 2017 aus. Sie zeigt, dass sich „Abgehängtsein“ im populärmedialen Diskurs in drei Narrative einordnen lässt:

a) ein infrastrukturelles, das ,von einer Schwäche öffentlicher und privater Infrastrukturen der Daseinsvorsorge insbesondere ländlicher Räume“ (Deppisch 2019, S. i) ausgeht,

b) ein wirtschaftliches, das sich auf Arbeitsplatzverluste und Abstiegsängste bezieht und

c) ein kulturelles, das sich in der Ablehnung moderner und kosmopolitischer Wertvorstellungen äußert.

Ob diese Narrative des Abgehängtseins spezifisch für ländliche Räume sind oder sich auf Deutschland insgesamt übertragen lassen, wurde dabei nicht untersucht. ${ }^{1}$

Die politische Aufmerksamkeit für ländliche Räume zeigt sich beispielsweise an der Besuchsreise, die Bundespräsident Steinmeier unter dem Titel „Land in Sicht Zukunft ländlicher Räume“" 2018/19 durchgeführt und auf seinen Webseiten herausgehoben dargestellt hat. ${ }^{2}$ Schon in seiner Antrittsrede als Bundespräsident hatte er 2017 gefragt: „Wie erhalten wir Hoffnung dort, wo im Dorf Schule, Arzt, Friseur, Tankstelle längst geschlossen sind und jetzt auch noch die letzte Busverbindung gekappt wird?“ (Steinmeier 2017, S. 6). In seiner Rede beim ,Zukunftsforum Ländliche Entwicklung“ wies er 2019 auf die Verantwortung des Staates hin und stellte die Rolle von Daseinsvorsorge heraus: „Der Staat muss also deutschlandweit eine gute Infrastruktur gewährleisten. Denn eine gute Infrastruktur ist mehr als Daseinsvorsorge, sie ist Dableibevorsorge. Darauf müssen sich die Menschen auch in ländlichen Gebieten verlassen können. Das ist der Staat ihnen schuldig!“ (Steinmeier 2019, S. 5).

Ein weiteres Beispiel für die politische Aufmerksamkeit ist die Kommission „Gleichwertige Lebensverhältnisse“, die 2018/19 mit Vertreter*innen aller Bundesministerien $^{3}$, der 16 Bundesländer und der kommunalen Spitzenverbände ${ }^{4}$ Vorschläge erarbeiten sollte, wie gleichwertige Lebensverhältnisse in Deutschland

\footnotetext{
1 Korte (2019, S. 434) konstatiert für die Gesellschaft in Deutschland: „Die Kluft zum entfesselten Tempo der Globalisierung speist unweigerlich die Sehnsucht nach dem Verlorenen. Zeitgleich wächst die Angst vor dem Verlust des gesellschaftlichen Status.“ Er kontrastiert einen „elitär wahrgenommenen kosmopolitischen Liberalismus“ mit einem ,neuen radikalen völkische[n] Autoritarismus“ (ebd., S. 431). Ein Vergleich der subjektiven Wahrnehmungen in unterschiedlichen Raum- und Siedlungstypen wäre hier ausgesprochen vielversprechend.

2 Siehe https://www.bundespraesident.de/DE/Bundespraesident/Land-in-Sicht/Land-in-Sicht-node.html, Zugriff: 24.10.2020.

3 Einschließlich der Beauftragten der Bundesregierung für Kultur und Medien, des Beauftragten der Bundesregierung für die neuen Länder und der Beauftragten für Migration, Flüchtlinge und Integration.

${ }^{4}$ Deutscher Landkreistag, Deutscher Städtetag, Deutscher Städte- und Gemeindebund.
} 
erreicht werden können. Nach dem Abschlussbericht der drei Vorsitzminister*innen bedeuten gleichwertige Lebensverhältnisse ,gute Entwicklungschancen und faire Teilhabemöglichkeiten für alle in Deutschland lebenden Menschen, unabhängig vom Wohnort" (BMI et al. 2019, S. 8). Auf diese Kommission werden wir noch einmal zurückkommen.

Bereits an diesen Beispielen wird die große Bedeutung von Daseinsvorsorge im Diskurs um gleichwertige Lebensverhältnisse - oft auf eine Gegenüberstellung von „Stadt“ und „Land“ verkürzt - deutlich, ebenso wie die Rolle des Staates für eine wie auch immer geartete Gewährleistung. Zugleich weist die Debatte zahlreiche Verkürzungen auf, wenn etwa ausschließlich von einem Abbau der Daseinsvorsorge die Rede ist, und wenn dies auf ,den“ ländlichen Raum bezogen wird. Beide Aspekte werden im Folgenden genauer betrachtet.

\section{Vielfalt ländlicher Räume}

Bis in die 1960er-Jahre war der ländliche Raum ,ein relativ homogener Raumtyp ..., der vor allem durch die große Bedeutung der Landwirtschaft, eine geringe Bevölkerungsdichte sowie die Dominanz bestimmter ländlicher Lebensweisen charakterisiert wurde“ (Mose 2018, S. 1324). Schon lange ist eine dichotome Raumbetrachtung von „Stadt“ und „Land“ der Realität nicht mehr angemessen. Stattdessen wird vielfach von einem Stadt-Land-Kontinuum ausgegangen und anerkannt, dass aufgrund der Vielfältigkeit ländlicher Räume von diesen im Plural und nicht im Singular gesprochen werden sollte. Ein typischer ländlicher Lebensstil existiert heute ebenso wenig wie eine typisch ländliche Wirtschaftsstruktur ${ }^{5}$ (Küpper und Milbert 2020).

Die eine, allseits anerkannte und verwendete Raumtypisierung für Deutschland ${ }^{6}$ gibt es nicht. Vielfach herangezogen werden die siedlungsstrukturelle Kreistypisierung des Bundesinstituts für Bau-, Stadt- und Raumforschung, die zwei städtische und zwei ländliche Regionstypen unterscheidet (BBSR 2012), und die Thünen-Typisierung ländlicher Räume, die vier ländliche und einen nicht-ländlichen Regionstyp beinhaltet (Küpper 2016; vgl. auch Küpper und Milbert 2020).

Die Thünen-Typisierung ländlicher Räume ist durch zwei Dimensionen gekennzeichnet: Für die Dimension Ländlichkeit wird aus fünf Indikatoren ein Index gebildet. Eine Region ist demnach umso ländlicher, je geringer die Siedlungsdichte, je höher der Anteil von Ein- und Zweifamilienhäusern und je höher der Anteil landund forstwirtschaftlicher Fläche ist und je geringer das regionale Bevölkerungspotenzial und die Erreichbarkeit der fünf nächsten Oberzentren sind (Küpper 2016). Die zweite Dimension bildet anhand von neun Indikatoren die sozioökonomische Lage ländlicher Räume ab. Als Ergebnis unterscheidet die Thünen-Typisierung flä-

\footnotetext{
5 Die wirtschaftliche Bedeutung von Land- und Forstwirtschaft sowie Fischerei in Deutschland ist auch in ländlichen Räumen im Durchschnitt sehr gering. So lag ihr Anteil 2017 bezogen auf die Erwerbstätigkeit in ländlichen Räumen (nach Thünen-Typisierung) bei 2,4\% (Deutschland insgesamt: 1,4\%) und bezogen auf die Bruttowertschöpfung bei 1,7\% (0,9\%) (eigene Berechnungen; Statistische Ämter des Bundes und der Länder 2020; Küpper 2016).

${ }^{6}$ Für internationale Vergleiche vgl. auch die Raumtypisierungen von OECD und Eurostat (Dijkstra und Poelman 2018).
} 
chendeckend für Deutschland auf Ebene der 361 Kreisregionen sehr ländliche, eher ländliche und nicht-ländliche Regionen. Die ländlichen Regionen sind zudem weiter unterteilt in solche mit guter und mit weniger guter sozioökonomischer Lage.

Das Thünen-Monitoring Ländliche Räume, in dessen Rahmen die Raumtypisierung erarbeitet wurde, zielt darauf ab, Lebensverhältnisse in ländlichen Räumen einerseits anhand von flächendeckend, in kleinräumiger Auflösung vorliegenden Daten der amtlichen Statistik und anhand von Ergebnissen aus Erreichbarkeitsanalysen darzustellen und andererseits die Sicht der Bevölkerung auf Ebene der Thünen-Typen ländlicher Räume durch repräsentative Befragungen einfließen zu lassen. Kernstück des Monitorings ist der Landatlas (www.landatlas.de; Steinführer et al. 2018), der über 80 Indikatoren kartographisch auf Kreisregions- oder Gemeindeverbandsebene abbildet.

Die Daten des Landatlasses zeigen: Ländliche Räume sind vielfältig, und sie sind keineswegs per se ,abgehängt“. Deutschland entwickelt sich räumlich auch nicht immer weiter auseinander, auch wenn dies oft behauptet wird (so z. B. Kersten et al. 2020). Küpper und Peters (2019) haben für 13 Indikatoren untersucht, wie sich regionale Disparitäten hinsichtlich Wirtschaftskraft, sozialer Lage sowie Daseinsvorsorge und Infrastruktur in Deutschland und seinen ländlichen Räumen seit 2000 entwickelt haben. Ihre Ergebnisse zeigen, dass ,,sich auf der räumlichen Analyseebene der Kreisregionen weder ein grundsätzliches Auseinanderdriften der Regionen in Deutschland noch eine pauschale Abkopplung ländlicher Räume von der gesamtgesellschaftlichen Entwicklung feststellen“ lässt (Küpper und Peters 2019, S. i; vgl. für ökonomische Indikatoren auch Röhl 2017; Braml und Felbermayr 2018; aus sozialgeographischer Perspektive: Miggelbrink 2020).

\section{Zusammenhänge zwischen Daseinsvorsorge und gleichwertigen Lebensverhältnissen}

Daseinsvorsorge ist ein Schlüsselbegriff des Sozialstaatsverständnisses der Bundesrepublik. Die Geschichte und Bedeutungsvielfalt des nicht unumstrittenen Begriffs kann im Rahmen dieses Beitrags nicht aufgearbeitet werden (vgl. verschiedene Beiträge in Neu 2009). In einem engeren Verständnis zählen zur Daseinsvorsorge „Dienstleistungen, an deren Angebot ein besonderes öffentliches Interesse besteht“" (Einig 2008, S. 17). Diese werden von öffentlichen, privaten und gemeinnützigen Akteuren sowie - nicht unbedeutend, wenn man etwa an soziale Dienste oder Freiwillige Feuerwehren denkt - ehrenamtlich von Privatpersonen erbracht. Jeder Daseinsvorsorgebereich (von der technischen Infrastruktur der Ver- und Entsorgung, der Kommunikationstechnik, den Verkehrsdiensten und der Energieversorgung über Einrichtungen der (vor)schulischen Bildung sowie der Aus- und Weiterbildung, kulturelle Einrichtungen, die Gesundheitsversorgung und Pflege, die Nahversorgung, die polizeiliche wie nichtpolizeiliche Gefahrenabwehr bis hin zur Wohnungsversorgung) ist durch eine komplexe Governancestruktur mit vielfältigen Akteuren und Regelungen charakterisiert sowie kontinuierlich im Wandel begriffen (Steinführer 2015). 
Wie die (konkrete) Ausgestaltung von Daseinsvorsorge vor dem Hintergrund des bundesdeutschen Sozialstaatsverständnisses ist auch die Gleichwertigkeit der Lebensverhältnisse ein wiederkehrendes Thema politischer und wissenschaftlicher Debatten in Deutschland. Diese lässt sich mit Aring (2010, S. 766) zum einen als die ,räumliche Ausprägung des Sozialstaats“ bezeichnen. Zum anderen aber bleibt die Formel von der Gleichwertigkeit der Lebensverhältnisse unbestimmt und je nach gesellschaftlicher Situation neu operationalisierbar. Diese Schwäche ist zugleich ihre Stärke - so setzen sich immer neue Generationen von Politiker*innen, Planer*innen und Wissenschaftler*innen mit dem Konzept bzw. normativen Ziel auseinander und versuchen, es mit Leben zu füllen und mit Daten zu untersetzen (Steinführer et al. 2020). Im Grundgesetz ist die „Herstellung gleichwertiger Lebensverhältnisse“" in Art. 72 genannt, allerdings nicht als Staatsziel, sondern zur Abgrenzung der Kompetenzen zwischen Bund und Ländern. Im Raumordnungsgesetz werden ,,ausgeglichene soziale, infrastrukturelle, wirtschaftliche, ökologische und kulturelle Verhältnisse“ als Grundsatz und Ziel der Raumordnung benannt. In der Umsetzung dessen kam der (öffentlichen) Daseinsvorsorge stets eine Schlüsselfunktion zu. „Nehmt euch die Stadt zum Bilde“ - so hieß es schon in den 1950erJahren, um mit einer ,sozialen Aufrüstung“ des Dorfes im Grunde eine Nivellierung von „Stadt“ und „Land“ zu erreichen (Fuchs 1996). Zwar waren diese Bemühungen noch nicht von einer Gleichwertigkeitsrhetorik begleitet (dies erfolgte erst mit dem fünften Raumordnungsbericht 1972 und dem Bundesraumordnungsprogramm von 1975), doch bildete Gleichwertigkeit bereits in den ersten beiden Nachkriegsjahrzehnten den impliziten Orientierungsrahmen der Raumordnungspolitik (Strubelt 2004, S. 251).

Als kleinster gemeinsamer Nenner lässt sich die öffentliche Daseinsvorsorge als Voraussetzung gleichwertiger Lebensverhältnisse identifizieren (so z. B. ARL 2006, 2016, Stielike 2010). Hier ist in den vergangenen Jahren eine Diskursverschiebung weg von einer Ausstattungs,,fixiertheit“ hin zu einer Ergebnisorientierung erfolgt: Demnach solle nicht das Vorhandensein einer Schule, sondern das Bildungsniveau der Bevölkerung einer Region der Maßstab von Chancengerechtigkeit im Sinne gleichwertiger Lebensverhältnisse sein (ARL 2016). Ein breiter Konsens besteht darüber, dass neben der Daseinsvorsorge auch Einkommens- und Erwerbsmöglichkeiten die Gleichwertigkeit der Lebensverhältnisse maßgeblich mitbestimmen. Zudem finden teilweise weitere Bereiche wie der Zustand der natürlichen Lebensgrundlagen Berücksichtigung in einschlägigen Indikatorensystemen oder auch im Leitfaden zur Durchführung des „Gleichwertigkeits-Checks“ bei Gesetzesvorhaben des Bundes (BMI 2020).

\section{Daseinsvorsorge in ländlichen Räumen: wo stehen wir?}

Für über 30 Einrichtungen der Daseinsvorsorge - z. B. verschiedene Ärztegruppen, Krankenhäuser, Schulen, Polizeistationen, Bushaltestellen - wurden für den Landatlas die Erreichbarkeiten zu Fuß, per Fahrrad und per Auto deutschlandweit auf $250 \mathrm{~m} \times 250 \mathrm{~m}$-Basis berechnet. Die Ergebnisse zeigen ein ähnliches Muster: Nahezu die gesamte automobile Bevölkerung in Deutschland kann innerhalb von $15 \mathrm{~min}$ 
Fahrzeit die betrachtete Einrichtung erreichen (vgl. z.B. Neumeier 2017, 2018a, 2018b). Die wenigen Prozent, die länger fahren müssen, wohnen in sehr dünn besiedelten, peripheren Regionen. Ist man jedoch auf das Fahrrad angewiesen oder muss zu Fuß gehen, reichen 15 min oftmals nicht aus. ${ }^{7}$ So können je nach ländlichem Raumtyp zwischen 75 und $91 \%$ der Bevölkerung den nächsten Hausarzt innerhalb von 15 min erreichen. Zu Fuß trifft dies für 47-65\% zu.

Die statistische Ermittlung potenzieller Erreichbarkeiten ist ein möglicher $\mathrm{Zu}$ gang, um die Daseinsvorsorgeausstattung etwa im Vergleich zwischen ländlichen und Ballungsräumen zu untersuchen. Ob sich aus erschwerter Erreichbarkeit im Alltag tatsächlich Probleme ergeben und welche sozialen Gruppen davon besonders betroffen sind, lässt sich nur durch Befragungen ermitteln. 2016 hat das Thünen-Institut für Ländliche Räume zwei standardisierte Repräsentativbefragungen der erwachsenen Bevölkerung bzw. von Familien mit mindestens einem Kind unter 13 Jahren in ländlichen Räumen durchgeführt. Beiden zufolge war der Großteil vorgegebener Einrichtungen (wie Grundschule, Gemeindeverwaltung oder Hausarzt/Hausärztin) im Umkreis von zehn Kilometern für die meisten Befragten (über 96\%) verfügbar. Bezogen auf die erwachsene Bevölkerung ( $n=1717$ Befragte) insgesamt waren kleine Lebensmittelläden (89\%), weiterführende Schulen $(87 \%)$ und spezialisierte Geschäfte etwa für Haushaltsgeräte und Möbel $(67 \%)$ am wenigsten verfügbar. Als Erschwernis für die Alltagsgestaltung wurde vorrangig das Fehlen letzterer genannt $(6 \%)$. Die Hälfte aller Familien $(n=956)$ verfügte wohnortnah über keine Einrichtung der Familienbildung oder eine entsprechende Beratungsstelle, als Alltagserschwernis wurde am häufigsten (von $8 \%$ ) ein fehlender Kinderarzt bzw. eine fehlende Kinderärztin genannt (vgl. auch Kreis 2021).

So vielfältig wie ländliche Räume sind, so unterschiedlich ist auch die Versorgung mit Daseinsvorsorgeeinrichtungen. Eine ausschließliche Abbau-Erzählung greift hier zu kurz - und wenn, dann reicht sie für Westdeutschland in die 1970er-Jahre zurück, als im Zuge der damaligen großflächigen Gebietsreformen zahlreiche Dorfschulen geschlossen und Gemeindeverwaltungen zentralisiert wurden. Abkopplungen vom Schienenverkehr, Ausdünnung der Busanbindungen und Schließung kleiner Nahversorger waren ebenfalls ein sich über Jahrzehnte ziehender Prozess. Für Ostdeutschland waren die Wiedervereinigung und das politische (wie wissenschaftliche) Paradigma der ,nachholenden Modernisierung“ entscheidende Anstöße für Daseinsvorsorgeverluste in ländlichen Räumen vor allem im Bereich der sozialen und medizinischen Versorgung.

Doch ist hier auch auf einen grundlegenden Ausbau vor allem technischer Infrastrukturen (von der Telefonversorgung über Straßenbau und Kanalisation bis hin zum Breitband) zu verweisen (Steinführer 2020). In Ost wie West schließlich wurde Daseinsvorsorge in den vergangenen Jahrzehnten nicht nur ab- und ausgebaut, sondern sie unterlag mit dem sich verändernden Sozialstaat und dem gewachsenen Einfluss europäischer Rechtsvorgaben einem grundlegenden Umbau. Liberalisierung, Privatisierung und teilweise Finanzialisierung (also die gewinnorientierte Veräußerung öffentlicher Daseinsvorsorge, z.B. kommunaler Wohnungsbestände,

\footnotetext{
${ }^{7}$ Berechnungen der Erreichbarkeit mit öffentlichen Verkehrsmitteln liegen bisher im Landatlas noch nicht vor, sind aber geplant.
} 
und deren Umwandlung in Anlageobjekte auf den Kapitalmärkten; Heeg 2013), aber auch privatwirtschaftliche Konzentrations- und Verdrängungsprozesse sind hier als Katalysatoren zu nennen. Das gilt z. B. für den Pflegesektor, Ver- und Entsorgungsinfrastrukturen und die Nahversorgung. Hinzu kommen in ländlichen Räumen grundlegende Veränderungen der räumlichen Verteilung von Daseinsvorsorgeeinrichtungen: Private wie öffentliche Anbieter konzentrieren sich, flankiert von landesund regionalplanerischen Zuweisungen zentralörtlicher Funktionen, zunehmend in Klein- und Mittelstädten und dort vor allem in deren Zentren. Gegeninitiativen wie etwa die Gründung und teilweise Förderung von Dorfläden oder Multifunktionshäusern vermögen hier nur bedingt Abhilfe zu schaffen.

\section{Wachsende Herausforderungen für die Daseinsvorsorge in ländlichen Räumen}

Ländliche Räume stehen vor wachsenden Herausforderungen. Zu nennen sind hier beispielsweise der demographische Wandel, die Digitalisierung von Wirtschaft und Gesellschaft, der Übergang zu einer nahezu treibhausgasneutralen Wirtschafts- und Lebensweise sowie sich ändernde Lebensstile, Erwerbsmuster und Lebensformen (vgl. Margarian und Weingarten 2019; Born und Steinführer 2018). Diese Herausforderungen betreffen in unterschiedlichem Maße auch die Daseinsvorsorge in ländlichen Räumen.

- Die Alterung der Bevölkerung, die eine bundesweit zu verzeichnende Entwicklung ist - von der aber periphere ländliche Räume besonders betroffen sind - und der in vielen Regionen seit langem zu verzeichnende Rückgang der Bevölkerung beeinflussen die Art und den Umfang der nachgefragten Leistungen, aber auch deren Finanzierbarkeit. So steigt mit zunehmender Alterung beispielsweise die Nachfrage nach medizinischer und pflegerischer Daseinsvorsorge. Die Finanzierbarkeit von Daseinsvorsorge ist durch eine Reduzierung der Bevölkerung doppelt betroffen (Küpper 2015): Zum einen verschlechtert sich bei sinkender Einwohnerzahl die Finanzsituation der betreffenden Kommune (weniger Steuereinnahmen, weniger Schlüsselzuweisungen, weniger Einnahmen an Gebühren). Zum anderen führen insbesondere bei leitungsgebundenen Infrastrukturen (Trinkwasser, Abwasser) hohe Fixkosten dazu, dass sich die Kosten pro Kopf erhöhen, wenn die Nutzerzahlen zurückgehen (Remanenzeffekt). Der demographische Wandel führt für viele Berufe und Regionen auch dazu, dass die Verfügbarkeit von Fachkräften sinkt. Hiervon sind auch die Erbringer von Daseinsvorsorgeleistungen, etwa im Pflegebereich, betroffen.

- Die Digitalisierung von Wirtschaft und Gesellschaft setzt den Zugang zu schnellem Breitband und leistungsfähige Mobilfunknetze voraus. Hier weisen noch einige, insbesondere ländliche Regionen weiße oder graue Flecken auf, wie viele in der Corona-Pandemie bei Homeoffice oder Homeschooling wieder schmerzhaft erfahren mussten. Die Digitalisierung bietet auch im Bereich der Daseinsvorsorge große Chancen. So kann die Telemedizin dazu beitragen, die Notwendigkeit von Fahrten zum nächsten, in Zukunft vielleicht weiter entfernten Arzt oder Kran- 
kenhaus zu verringern. Autonomes Fahren könnte die Mobilität vieler Menschen erhöhen, was insbesondere in dünn besiedelten Regionen mit langen Wegen von großer Bedeutung wäre.

- Der Übergang zu einer nahezu treibhausgasneutralen Wirtschafts- und Lebensweise, der bis 2050 erforderlich ist, damit Deutschland seine Klimaziele erreichen kann, wird unter anderem dazu führen, dass Verkehrsmobilität teurer wird. Hiervon werden die Menschen in ländlichen Räumen stärker betroffen sein als in Großstädten. Wie die Erreichbarkeitsanalysen des Landatlasses zeigen, sind die Wege zum nächsten Arzt, zum Discounter, zur Schule oder zur Polizei in ländlichen Räumen in der Regel länger.

- Sich ändernde Lebensstile, Erwerbsmuster und Lebensformen betreffen auch die Daseinsvorsorge. Ein prominentes Beispiel hierfür ist die gestiegene Nachfrage nach Kinderbetreuung und der in den letzten Jahren erfolgte Ausbau von Kitas und Ganztagsbetreuung in Schulen. Dies ist vor dem Hintergrund veränderter Erwerbsmuster von Eltern vor allem in den westlichen Bundesländern und dabei insbesondere von Müttern kleinerer Kinder zu sehen (Born und Steinführer 2018, S. 34). Das Beispiel verweist auch darauf, wie gesellschaftlicher Wandel wiederum Erwartungen an Daseinsvorsorge und die Rolle des Staates für deren Bereitstellung innerhalb kurzer Zeit grundlegend verändern kann. Ähnliches lässt sich aus der Corona-Pandemie für den Gesundheitssektor erwarten.

\section{Politische Dauer- und Querschnittsaufgabe}

Wie kann vor dem Hintergrund der genannten Herausforderungen die Daseinsvorsorge in ländlichen Räumen auch zukünftig gesichert werden? (Wie) können gleichwertige Lebensverhältnisse in allen Regionen Deutschlands erreicht werden? Diese Fragen werden seit den 1970er-Jahren immer wieder gestellt, und sie können in dem vorliegenden Beitrag grundsätzlich nicht beantwortet werden - denn dies ist zuallererst eine politische Gestaltungsfrage. Stattdessen greifen wir hier an einzelnen Beispielen auf, wie diese Fragen in jüngster Zeit politisch adressiert wurden.

Gleichwertige Lebensverhältnisse ,herzustellen und dabei sozial, ökonomisch und ökologisch ausgewogen ... zu gestalten, bleibt eine Aufgabe für die kommende Dekade“, wie die Bundesregierung insbesondere mit Bezug auf ländliche Räume jüngst im „Dritten Bericht der Bundesregierung zur Entwicklung der ländlichen Räume“ (BMEL 2020) betonte. Es ist keine neue Aufgabe, sondern eine Daueraufgabe. In einem föderalen Staat wie Deutschland ist es zugleich eine Querschnittsaufgabe, die Bund, Länder und Kommunen betrifft, und auf diesen Ebenen jeweils viele Ressorts.

Deshalb war es zu begrüßen, dass sich Vertreter*innen der genannten drei Ebenen im September 2018 zur Kommission Gleichwertige Lebensverhältnisse konstituierten. Hohe Erwartungen wurden hiermit verbunden. Dass diese nicht erfüllt wurden, zeigte sich im Juli 2019 schon daran, dass es nicht gelungen war, sich auf gemeinsame Schlussfolgerungen zu einigen. Stattdessen legten die drei Vorsitzminister*innen Seehofer, Klöckner und Giffey mit „Unser Plan für Deutschland - Gleichwertige Lebensverhältnisse überall“ (BMI 2019) ihre eigenen Schlussfolgerungen vor. Zu 
deren Umsetzung beschloss die Bundesregierung zwölf Maßnahmen (N. N. 2019). Einzelne davon adressieren explizit Bereiche der Daseinsvorsorge. ${ }^{8}$ Andere Maßnahmen, wie die gezielte Förderung strukturschwacher Regionen durch ein (sich aus bestehenden Förderprogrammen bzw. -maßnahmen speisendes) ,neues gesamtdeutsches Fördersystem“ lassen auch positive Auswirkungen auf die Daseinsvorsorge in ländlichen Räumen erwarten. Das ,neue gesamtdeutsche Fördersystem“ bietet die Chance, dass Fördermaßnahmen unterschiedlicher Ressorts stärker aufeinander abgestimmt werden. Ob diese Chance genutzt wird oder ob es faktisch eher nur zu einem gemeinsamen Berichtswesen kommt, wird die Zukunft zeigen. Den Querschnittscharakter des Gleichwertigkeitsziels greift auch die Maßnahme „Gleichwertige Lebensverhältnisse als Richtschnur setzen“ auf. Im Ergebnis der Kommissionsarbeit führte der Bund 2020 einen „Gleichwertigkeits-Check“ ein. Demnach ist im Gesetzgebungsverfahren zu prüfen, ,ob und wie sich Gesetzesvorhaben des Bundes auf die Gleichwertigkeit der Lebensverhältnisse der Menschen auswirken“ (BMI 2020: 1). ${ }^{9}$ Es bleibt abzuwarten, ob dies zu einer wünschenswerten besseren Abwägung von Gesetzesfolgen beiträgt oder ob der Gleichwertigkeits-Check ein Papiertiger werden wird (für einen ähnlichen Ansatz, das ,rural proofing“ in England, vgl. Atterton 2008). Abzuwarten bleibt auch, zu welchen konkreten Ergebnissen der Folgeprozess ${ }^{10}$ zur Kommission Gleichwertige Lebensverhältnisse führen wird. Positiv ist in jedem Fall, dass gleichwertige Lebensverhältnisse auf der politischen Agenda weiter nach oben gerückt sind.

Auch auf der Länderebene hat der Themenkomplex „Daseinsvorsorge - gleichwertige Lebensverhältnisse - ländliche Räume“ in den letzten Jahren an politischer Bedeutung gewonnen. Dies zeigt sich beispielsweise darin, dass der bayerische Landtag eine Enquete-Kommission „Gleichwertige Lebensverhältnisse in Bayern“ eingesetzt hatte, deren Bericht im Landtag 2018 diskutiert wurde (Bayerischer Landtag o.J.). Ein anderes Beispiel ist, dass der Landtag in Thüringen derzeit über einen Gesetzesentwurf diskutiert, der unter anderem vorsieht, die Herstellung gleichwertiger Lebensverhältnisse als Staatsziel in die Verfassung Thüringens aufzunehmen (Thüringer Landtag 2020), ähnlich wie dies in Baden-Württemberg, Bayern, Brandenburg und Hessen der Fall ist.

Für die meisten Bereiche der Daseinsvorsorge und damit eine lebensweltlich erfahrbare Gleichwertigkeit von Lebensverhältnissen sind jedoch nicht der Bund oder die Länder zuständig. Vielmehr sind die Kommunen die „Gestalter der konkreten, regional spezifischen Herausforderungen der Daseinsvorsorge“ (Winkler-Kühlken 2019, S. 24). Der Handlungs- und Gestaltungsspielraum vieler Kommunen bei der Erfüllung ihrer Pflichtaufgaben, insbesondere aber bei freiwilligen Aufgaben, leidet oftmals unter fehlenden finanziellen (und personellen) Ressourcen. Zwar unterstüt-

\footnotetext{
8 Siehe Maßnahme 3 „Breitband und Mobilfunk flächendeckend ausbauen“, 4 ,Mobilität und Verkehrsinfrastruktur in der Fläche verbessern“ und 9 „Qualität und Teilhabe in der Kindertagesbetreuung sichern“.

9 Beim Gleichwertigkeits-Check handelt sich nicht um eine explizit in § 44 GGO genannte Gesetzesfolge. Er hat appellativen Charakter (BMI 2020).

$10 \mathrm{Zu}$ nennen sind hier insbesondere die Einsetzung eines Ausschusses auf Staatssekretärsebene zur Steuerung und zum Nachhalten der Umsetzung der zwölf Maßnahmen und die gesonderte Erörterung der Maßnahmen mit den Ländern und den kommunalen Spitzenverbänden.
} 
zen der Bund und die Länder Kommunen bei der Gewährleistung der Daseinsvorsorge auch mit einer Vielzahl von Förderprogrammen und -maßnahmen. Genannt seien hier zum Beispiel die im neuen gesamtdeutschen Fördersystem zusammengefassten Förderaktivitäten und die landesspezifischen „Programme zur Entwicklung des ländlichen Raums“. So sinnvoll dies im Einzelfall ist, darf dies den Blick darauf nicht verstellen, dass auf manche Förderaktivitäten verzichtet werden könnte, wenn die Kommunen grundsätzlich finanziell und personell besser ausgestattet wären. Der Sachverständigenrat für Ländliche Entwicklung beim BMEL empfiehlt daher, „eine Verbesserung der Steuerausstattung der Kommunen herbeizuführen, um den berechtigten Anforderungen der Bevölkerung in ländlichen Räumen an die Daseinsvorsorge Rechnung zu tragen“ (SRLE 2017, S. 10; ähnlich: Facharbeitsgruppe 3 „Raumordnung und Statistik“ 2019).

Handlungs- und Gestaltungsspielräume könnten auch dadurch vergrößert werden, dass bestehende rechtliche Regelungen im Bereich der Daseinsvorsorge hinterfragt und ggf. angepasst werden, um flexible, adäquate Lösungen zu ermöglichen (SRLE 2017; zur Diskussion über die Flexibilisierung von Standards vgl. Winkler-Kühlken 2019 sowie Winkel 2010). Dies wäre insbesondere für von starkem Bevölkerungsrückgang und Alterung besonders betroffene Regionen wichtig, in denen nicht zuletzt ein „Management von Schrumpfung“ (BMELV 2007, S. 3; vgl. Küpper et al. 2013) erforderlich ist.

\section{Fazit}

Daseinsvorsorge ist ,,von zentraler Bedeutung für die Verwirklichung von Lebenschancen, die Teilhabe am gesellschaftlichen Leben sowie für die Alltagsgestaltung und die Lebensqualität der Einwohner" (SRLE 2017, S. 9). Sie ist damit ein zentraler Bestandteil gleichwertiger Lebensverhältnisse. Zugleich ist sie ,kein statisches Konzept und kein fester Katalog - was genau sie umfasst, welche Erwartungen an sie herangetragen werden, welche Steuerungsmodelle zum Einsatz kommen und wie sie gewährleistet werden kann, verändert sich kontinuierlich mit dem gesellschaftlichen Wandel“" (Steinführer und Küpper 2020, S. 24). Die Gewährleistung der Daseinsvorsorge zu angemessenen Preisen und in zumutbarer Entfernung stellt daher eine Daueraufgabe dar, die mit Aus-, Ab- und Umbau einhergeht. Sie wird von sozialem, ökonomischem und technologischem Wandel beeinflusst und wirkt selbst auf diese Prozesse ein. Sie ist zudem eine Querschnittsaufgabe für Bund, Länder und insbesondere Kommunen. Nicht zuletzt ist sie eine flächendeckende Aufgabe, stellt aber insbesondere wirtschaftlich strukturschwache, von Alterung und Bevölkerungsrückgang betroffene und dünn besiedelte Regionen vor besondere Herausforderungen. Gleichwohl stehen auch wachsende und wirtschaftlich prosperierende ländliche und nicht-ländliche Räume vor Anpassungs- und Umbauerfordernissen der Daseinsvorsorge. Eine verkürzte Vorstellung von „dem“ ländlichen Raum, der ausschließlich strukturschwach, peripher und ,abgehängt“ sei und in dem die Daseinsvorsorge immer schlechter werde, geht an der Vielfalt ländlicher Räume und der Lebenswirklichkeit vieler Bewohner*innen vorbei. 
Funding Open Access funding enabled and organized by Projekt DEAL.

Open Access Dieser Artikel wird unter der Creative Commons Namensnennung 4.0 International Lizenz veröffentlicht, welche die Nutzung, Vervielfältigung, Bearbeitung, Verbreitung und Wiedergabe in jeglichem Medium und Format erlaubt, sofern Sie den/die ursprünglichen Autor(en) und die Quelle ordnungsgemäß nennen, einen Link zur Creative Commons Lizenz beifügen und angeben, ob Änderungen vorgenommen wurden.

Die in diesem Artikel enthaltenen Bilder und sonstiges Drittmaterial unterliegen ebenfalls der genannten Creative Commons Lizenz, sofern sich aus der Abbildungslegende nichts anderes ergibt. Sofern das betreffende Material nicht unter der genannten Creative Commons Lizenz steht und die betreffende Handlung nicht nach gesetzlichen Vorschriften erlaubt ist, ist für die oben aufgeführten Weiterverwendungen des Materials die Einwilligung des jeweiligen Rechteinhabers einzuholen.

Weitere Details zur Lizenz entnehmen Sie bitte der Lizenzinformation auf http://creativecommons.org/ licenses/by/4.0/deed.de.

\section{Literatur}

Akademie für Raumordnung und Landesplanung (Hrsg.). 2006. Gleichwertige Lebensverhältnisse: eine wichtige gesellschaftspolitische Aufgabe neu interpretieren! ARL-Positionspapier 69. Hannover: ARL. Erarbeitet vom Ad-hoc-Arbeitskreis „Gleichwertigkeit der Lebensverhältnisse“.

Akademie für Raumordnung und Landesplanung (Hrsg.). 2016. Daseinsvorsorge und gleichwertige Lebensverhältnisse neu denken. Perspektiven und Handlungsfelder. ARL-Positionspapier 108. Hannover: ARL. Erarbeitet vom Ad-hoc-Arbeitskreis „Daseinsvorsorge und gleichwertige Lebensbedingungen“.

Aring, Jürgen. 2010. Gleichwertige Lebensverhältnisse - Inverse frontiers - Selbstverantwortungsräume. In Weniger ist Zukunft. 19 Städte - 19 Themen, Hrsg. IBA Stadtumbau Sachsen-Anhalt, 764-777. Berlin: Jovis.

Atterton, Jane. 2008. Rural proofing in England: a formal commitment in need of review. Discussion Paper Series 20. Newcastle upon Tyne: Centre for Rural Economy.

Bayerischer Landtag. o.J. Bericht der Enquete-Kommission „Gleichwertige Lebensverhältnisse in ganz Bayern". Drucksache 17/19700.

BBSR [Bundesinstitut für Bau-, Stadt- und Raumforschung] (Hrsg.). 2012. Raumabgrenzungen und Raumtypen des BBSR. Analysen Bau.Stadt.Raum 6. Stuttgart: Steiner.

BMEL [Bundesministerium für Ernährung und Landwirtschaft] (Hrsg.). 2020. Entwicklung ländlicher Räume: Dritter Bericht der Bundesregierung. Berlin: BMEL.

BMI [Bundesministerium des Inneren, für Bau und Heimat]. 2020. Leitfaden zur Durchführung des „Gleichwertigkeits-Checks“ (GL-Check) bei Gesetzesvorhaben des Bundes. https://www.bmi. bund.de/SharedDocs/downloads/DE/veroeffentlichungen/themen/heimat-integration/gleichwertigelebensverhaeltnisse/gleichwertigkeits-check.pdf. Zugegriffen: 13. Aug. 2020.

BMI [Bundesministerium des Innern, für Bau und Heimat], BMEL [Bundesministerium für Ernährung und Landwirtschaft] und BMFSFJ [Bundesministerium für Familie, Senioren, Frauen und Jugend]. 2019. Unser Plan für Deutschland - Gleichwertige Lebensverhältnisse überall. Berlin: BMI, BMEL, BMFSFJ.

Born, Karl Martin, und Annett Steinführer. 2018. Ländliche Räume: Definitionsprobleme, Herausforderungen und gesellschaftlicher Wandel. In Kompendium Jugend im ländlichen Raum, Hrsg. Margit Stein, Lukas Scherak, 17-44. Bad Heilbrunn: Julius Klinkhardt.

Braml, Martin, und Gabriel Felbermayr. 2018. Regionale Ungleichheit in Deutschland und der EU: Was sagen die Daten? ifo Schnelldienst 71(7):37-49.

Bundesministerium für Ernährung, Landwirtschaft und Verbraucherschutz (Hrsg.). 2007. Politik für ländliche Räume: Konzeption zur Weiterentwicklung der Politik für ländliche Räume. Bonn: BMELV.

Deppisch, Larissa. 2019. „, Wo sich Menschen auf dem Land abgehängt fühlen, hat der Populismus freie Bahn" - eine Analyse des populär-medialen Diskurses zu der Bedeutung von Infrastrukturverfall, Abstiegsangst und rechten (extremistischen) Werten für den Zuspruch zum Rechtspopulismus. Thünen Working Paper 119. Braunschweig: Johann Heinrich von Thünen-Institut. 
Dijkstra, Lewis, und Hugo Poelman. 2018. Regional typologies overview. [Brussels]: European Commission, DG Regio, 12 S. https://ec.europa.eu/eurostat/statistics-explained/pdfscache/11609.pdf. Zugegriffen: 11. Nov. 2020.

Ehrenstein, Claudia. 2016. Zukunftsfragen entscheiden sich auf dem Lande. Die Welt, 15. November 2016.

Einig, Klaus. 2008. Regulierung der Daseinsvorsorge als Aufgabe der Raumordnung im Gewährleistungsstaat. Informationen zur Raumentwicklung (1/2):17-40.

Facharbeitsgruppe 3 ,Raumordnung und Statistik“. 2019. Bericht der Facharbeitsgruppe 3 ,Raumordnung und Statistik“. In Unser Plan für Deutschland - Gleichwertige Lebensverhältnisse überall, Hrsg. BMI [Bundesministerium des Innern, für Bau und Heimat], BMEL [Bundesministerium für Ernährung und Landwirtschaft] und BMFSFJ [Bundesministerium für Familie, Senioren, Frauen und Jugend], 51-77. Berlin: BMI, BMEL, BMFSFJ.

Fuchs, Thomas. 1996. Macht Euch die Stadt zum Bilde! Über die Modernisierung des ländlichen Raumes. Stadt, Raum und Gesellschaft 4. Pfaffenweiler: Centaurus.

Heeg, Susanne. 2013. Wohnungen als Finanzanlage. Auswirkungen von Responsibilisierung und Finanzialisierung im Bereich des Wohnens. sub\urban - Zeitschrift für kritische Stadtforschung 1(1):75-99.

Kersten, Jens, Claudia Neu, und Berthold Vogel. 2020. Gleichwertigkeit der Lebensverhältnisse - für eine Politik des Zusammenhalts. Ländlicher Raum 71(3):6-11.

Korte, Hans-Rudolf. 2019. Gestaltungsmacht für Gemeinwohl: Zur Rolle des Bundespräsidenten. Zeitschrift für Politikwissenschaft 29(3):431-437.

Kreis, Joachim. 2021. Leben in ländlichen Räumen. Ausgewählte Ergebnisse zweier Bevölkerungsbefragungen. Thünen Report. Braunschweig: Johann Heinrich von Thünen-Institut. Im Erscheinen.

Küpper, Patrick. 2015. Auswirkungen der demografischen Entwicklungen auf die Daseinsvorsorge: Regionale Betroffenheit und kommunale Gestaltungsmöglichkeiten. KommunalPraxis Spezial 15(1):23-28.

Küpper, Patrick. 2016. Abgrenzung und Typisierung ländlicher Räume. Thünen Working Paper 68. Braunschweig: Johann Heinrich von Thünen-Institut.

Küpper, Patrick, und Jan Cornelius Peters. 2019. Entwicklung regionaler Disparitäten hinsichtlich Wirtschaftskraft, sozialer Lage sowie Daseinsvorsorge und Infrastruktur in Deutschland und seinen ländlichen Räumen. Thünen Report 66. Braunschweig: Johann Heinrich von Thünen-Institut.

Küpper, Patrick, und Antonia Milbert. 2020. Typen ländlicher Räume in Deutschland. In Land in Sicht. Ländliche Räume in Deutschland zwischen Prosperität und Peripherisierung Schriftenreihe 10362, Hrsg. Christian Krajewski, Claus-C. Wiegandt, 82-97. Bonn: Bundeszentrale für politische Bildung.

Küpper, Patrick, Annett Steinführer, Steffen Ortwein, und Moritz Kirchesch. 2013. Regionale Schrumpfung gestalten: Handlungsspielräume zur langfristigen Sicherung gesellschaftlicher Teilhabe schaffen und nutzen. Braunschweig: Johann Heinrich von Thünen-Institut.

Margarian, Anne, und Peter Weingarten. 2019. Wirtschaftsentwicklung in ländlichen Räumen - aktuelle und künftige Einflussfaktoren. Landentwicklung aktuell 24:68-70.

Miggelbrink, Judith. 2020. Ländliche Räume - strukturschwach, peripher, abgehängt? In Land in Sicht. Ländliche Räume in Deutschland zwischen Prosperität und Peripherisierung Schriftenreihe 10362, Hrsg. Christian Krajewski, Claus-C. Wiegandt, 64-79. Bonn: Bundeszentrale für politische Bildung.

Mose, Ingo. 2018. Ländliche Räume. In Handwörterbuch der Stadt- und Raumentwicklung, Hrsg. Akademie für Raumforschung und Landesplanung, 1323-1334. Hannover: ARL.

N.N. 2019. Maßnahmen der Bundesregierung zur Umsetzung der Ergebnisse der Kommission „Gleichwertige Lebensverhältnisse“. https://www.bmi.bund.de/SharedDocs/downloads/DE/veroeffentlichungen/ themen/heimat-integration/gleichwertige-lebensverhaeltnisse/kom-gl-massnahmen.pdf?_blob= publicationFile\&v=4. Letzter Zugriff: 10. Nov. 2020.

Neu, Claudia (Hrsg.). 2009. Daseinsvorsorge. Eine gesellschaftswissenschaftliche Annäherung. Wiesbaden: VS.

Neumeier, Stefan. 2017. Regionale Erreichbarkeit von ausgewählten Fachärzten, Apotheken, ambulanten Pflegediensten und weiteren ausgewählten Medizindienstleistungen in Deutschland - Abschätzung auf Basis des Thünen-Erreichbarkeitsmodells. Thünen Working Paper 77. Braunschweig: Johann Heinrich von Thünen-Institut.

Neumeier, Stefan. 2018a. Erreichbarkeit durch die Polizei - Kennzahlen und Karten für Deutschland, basierend auf einer kleinräumigen, flächendeckenden GIS-Erreichbarkeitsanalyse. Kartographische Nachrichten 68(4):192-201.

Neumeier, Stefan. 2018b. Erreichbarkeit von Regelschulen in Deutschland: eine Betrachtung des ersten Bildungswegs nach Primarstufe, Sekundarstufe 1 und Sekundarstufe 2. Thünen Working Paper 113. Braunschweig: Johann Heinrich von Thünen-Institut. 
Reuters. 2018. Forschungsministerin - 5G nicht an jeder Milchkanne erforderlich. https://de.reuters.com/ article/deutschland-bundesnetzagentur-5g-idDEKCN1NQ1MU. Zugegriffen: 12. Nov. 2020.

Röhl, Klaus-Heiner. 2017. Regionale Konvergenzprozesse in Deutschland: Der ländliche Raum holt auf. IW-Report 28/2017. Köln: Institut der deutschen Wirtschaft.

SRLE [Sachverständigenrat Ländliche Entwicklung]. 2017. Weiterentwicklung der Politik für ländliche Räume in der 19. Legislaturperiode: Stellungnahme des Sachverständigenrats Ländliche Entwicklung (SRLE) beim Bundesministerium für Ernährung und Landwirtschaft (BMEL). Berlin: SRLE.

Statistische Ämter des Bundes und der Länder. Regionaldatenbank Deutschland, Code 13312-01-05-4, Code 82111-01-05-4. https://www.regionalstatistik.de/genesis/online. Zugegriffen: 12. Nov. 2020.

Steinführer, Annett. 2015. Bürger in der Verantwortung. Veränderte Akteursrollen in der Bereitstellung ländlicher Daseinsvorsorge. Raumforschung und Raumordnung 73(1):5-16.

Steinführer, Annett. 2020. Daseinsvorsorge in ländlichen Räumen. Zwischen Abbau, Umbau und Ausbau. In: Regionalentwicklung in Ostdeutschland. Dynamiken, Perspektiven und der Beitrag der Humangeographie. Hrsg. Sören Becker und Matthias Naumann, 375-387. Berlin: Springer Spektrum.

Steinführer, Annett, und Patrick Küpper. 2020. Daseinsvorsorge in ländlichen Räumen. Informationen zur politischen Bildung 343(2):16-25.

Steinführer, Annett, Torsten Osigus, Patrick Küpper, Stefan Neumeier, und Joachim Kreis. 2018. Ländliche Räume im Fokus: der Landatlas als neue Informationsplattform. In Flächennutzungsmonitoring $X$. Flächenpolitik - Flächenmanagement - Indikatoren IÖR Schriften 76, Hrsg. Gotthard Meinel, Ulrich Schumacher, Martin Behnisch, und Tobias Krüger, 153-160. Berlin: Rhombos.

Steinführer, Annett, Christian Hundt, Patrick Küpper, Anne Margarian, und Peter Mehl. 2020. Gleichwertigkeit der Lebensverhältnisse - wissenschaftliche Verständnisse und Zugänge. Ländlicher Raum 71(3):12-17.

Steinmeier, Frank-Walter. 2017. Bundespräsident Frank-Walter Steinmeier nach seiner Vereidigung zum Bundespräsidenten im Deutschen Bundestag am 22. März 2017 in Berlin, 9 S. https:// www.bundespraesident.de/SharedDocs/Downloads/DE/Reden/2017/03/170322-Vereidigung.pdf? blob=publicationFile. Zugegriffen: 11. Nov. 2020.

Steinmeier, Frank-Walter. 2019. Bundespräsident Frank-Walter Steinmeier beim „Zukunftsforum Ländliche Entwicklung " auf der Internationalen Grünen Woche am 23. Januar 2019 in Berlin, 6 S. https://www.bundespraesident.de/SharedDocs/Downloads/DE/Reden/2019/01/190123-GrueneWoche-Zukunftsforum.pdf?_blob=publicationFile. Zugegriffen: 11. Nov. 2020.

Stielike, Jan Matthias. 2010. Wieviel Gleichheit erfordert Gleichwertigkeit? Folgerungen einer Neuinterpretation des Gleichwertigkeitsziels für die Daseinsvorsorge. In Jahrbuch StadtRegion 2009/2010 Schwerpunkt: Stadtkultur und Kreativität, Hrsg. Christine Hannemann, Herbert Glasauer, Jörg Pohlan, Andreas Pott, und Volker Kirchberg, 129-140. Opladen, Farmington Hills: Barbara Budrich.

Strotdrees, Gisbert. 2018. Zwischen Landlust und Randfrust - mediale Dorf-Bilder der Gegenwart. Zeitschrift für Agrargeschichte und Agrarsoziologie 66(2):41-53.

Strubelt, Wendelin. 2004. Gleichwertigkeit der Lebensverhältnisse als Element der sozialen Integration. In Angewandte Soziologie, Hrsg. Robert Kecskes, Michael Wagner, und Christof Wolf, 247-283. Wiesbaden: VS.

Thüringer Landtag. 2020. Gesetzentwurf der Fraktion der CDU: Fünftes Gesetz zur Änderung der Verfassung des Freistaats Thüringen - Aufnahme von Staatszielen und Stärkung von Gleichheitsrechten. Drucksache 7/1629 vom 23.09.2020.

Volkert, Lilith; Datli, Baran (2017): Fünf Gründe, warum die AfD in Sachsen stärkste Kraft geworden ist. Süddeutsche Zeitung, 25. September 2017.

Winkel, Rainer. 2010. Mindeststandards - aber welche. PlanerIn (5):39-41.

Winkler-Kühlken, Bärbel. 2019. Standards in der Raumordnung auf dem Prüfstand. Aus Politik und Zeitgeschichte 69(46):19-25. 